\title{
GENERALIZED COMPLEX FUZZY AND ANTI-FUZZY $H_{v}$-SUBGROUPS
}

\author{
M. AL TAHAN ${ }^{1}$ AND B. DAVVAZ ${ }^{2, *}$ \\ ${ }^{1}$ Department of Mathematics, Lebanese International University, Lebanon \\ ${ }^{2}$ Department of Mathematics, Yazd University, Yazd, Iran \\ *Corresponding author: davvaz@yazd.ac.ir
}

\begin{abstract}
In this paper, we introduce the concept of generalized complex fuzzy subhypergroup $\left(H_{v}\right.$ subgroup) as well as the generalized concept of complex anti-fuzzy subhypergroup ( $H_{v}$-subgroup). We investigate their properties and their relations with the generalized traditional fuzzy (anti-fuzzy) subhypergroup ( $H_{v}$-subgroup).
\end{abstract}

\section{IntRoduction}

Hyperstructure theory was born in 1934, when Marty [8] gave the definition of hypergroup as a natural generalization of the concept of group based on the notion of hyperoperation at the eighth Congress of Scandinavian Mathematicians. He analyzed their properties and applied them to groups, illustrated some applications and showed its utility in the study of groups, algebraic functions and relational fractions. Recently, the hypergroups are studied from the theoretical point of view and for their applications to many subjects of pure and applied mathematics: geometry, topology, cryptography and code theory, graphs and hypergraphs, probability theory, binary relations, theory of fuzzy and rough sets, automata theory, economy, etc. (see $[4,6]$ ). A hypergroup is an algebraic structure similar to a group, but the composition of two elements is a non-empty set. On the other hand, the fuzzy mathematics forms a branch of mathematics related to fuzzy set theory and fuzzy logic. It was introduced in 1965 after the publication of L. A. Zadeh

Received 2017-10-30; accepted 2018-01-11; published 2018-09-05.

2010 Mathematics Subject Classification. 20N20, 20N25, $03 \mathrm{E} 72$.

Key words and phrases. hypergroup; complex fuzzy set; generalized fuzzy subhypergroup.

(C) 2018 Authors retain the copyrights of their papers, and all open access articles are distributed under the terms of the Creative Commons Attribution License. 
(see [15]), who is considered as the pioneer of this theory, as an extension of the classical notion of set, when he proposed the idea of a multi-valued logic, which extends the traditional concept of a bivalent logic, which becomes a particular case of the new theory. The fuzzy set theory is based on the principle called by L. A. Zadeh "the principle of incompatibility", that is "the closer a phenomenon is studied, the more indistinct its definition becomes". Fuzzy sets are sets whose elements have degrees of membership. In classical set theory, the membership of elements in a set is assessed in binary terms according to a bivalent condition an element either belongs or does not belong to the set. By contrast, fuzzy set theory permits the gradual assessment of the membership of elements in a set; this is described with the aid of a membership function valued in the real unit interval $[0,1]$. Fuzzy sets generalize classical sets, since the indicator functions of classical sets are special cases of the membership functions of fuzzy sets, if the latter only take values 0 or 1. Many researchers worked on fuzzy set theory, its applications and its extensions. An important extension of this theory was proposed by Raymot et al. [10,11]. They introduced the concept of complex fuzzy sets in which the codomain of membership function was the unit disc of the complex plane. Then they defined different fuzzy complex operations and relations such as the complement of a complex fuzzy set, intersection and union of complex fuzzy sets.

Davvaz et al. (see [7]) introduced the concept of generalized traditional fuzzy $H_{v}$-subgroups and the authors in [1] introduced the concept of complex fuzzy and anti-fuzzy $H_{v}$-subgroups. Our paper extends their results to complex fuzzy sets, and it is constructed as follows: after an Introduction, in Section 2 we present some definitions and results about hyperstructures and traditional fuzzy subhyperstructures. In Section 3, we present the results of generalized fuzzy $H_{v}$-subgroups and introduce the concept of generalized anti-fuzzy $H_{v^{-}}$-subgroups. In Section 4 , we extend the definitions of generalized fuzzy and anti-fuzzy $H_{v^{-}}$ subgroups and define generalized complex fuzzy and anti-fuzzy $H_{v}$-subgroups. We investigate their properties and present different examples on them.

\section{Preliminaries}

In this section, we present some definitions and theorems related to hyperstructures and fuzzy subhyperstructures that are used throughout the paper.

Definition 2.1. Let $H$ be a non-empty set. Then, a mapping $\circ: H \times H \rightarrow \mathcal{P}^{*}(H)$ is called a binary hyperoperation on $H$, where $\mathcal{P}^{*}(H)$ is the family of all non-empty subsets of $H$. The couple $(H, \circ)$ is called a hypergroupoid.

In the above definition, if $A$ and $B$ are two non-empty subsets of $H$ and $x \in H$, then we define:

$$
A \circ B=\bigcup_{\substack{a \in A \\ b \in B}} a \circ b, x \circ A=\{x\} \circ A \text { and } A \circ x=A \circ\{x\} .
$$


Definition 2.2. A hypergroupoid $(H, \circ)$ is called a:

- semihypergroup if for every $x, y, z \in H$, we have $x \circ(y \circ z)=(x \circ y) \circ z$;

- quasihypergroup if for every $x \in H, x \circ H=H=H \circ x$ (This condition is called the reproduction axiom);

- hypergroup if it is a semihypergroup and a quasihypergroup;

- $H_{v}$-group if it is a quasihypergroup and for every $x, y, z \in H$, we have $x \circ(y \circ z) \cap(x \circ y) \circ z \neq \emptyset$.

Definition 2.3. Let $(H, \circ)$ be a hypergroup (or $H_{v}$-group) and $K \subseteq H$. Then $(K, \circ)$ is a subhypergroup (or $H_{v}$-subgroup) of $(H, \circ)$ if for all $a \in K$, we have that $a \circ K=K \circ a=K$.

Definition 2.4. [15] A fuzzy set, defined on a universe of discourse $U$ is characterized by a membership function $\mu_{A}(x)$ that assigns any element a grade of membership in A. The fuzzy set may be represented by the set of ordered pairs $A=\left\{\left(x, \mu_{A}(x)\right): x \in U\right\}$, where $\mu_{A}(x) \in[0,1]$.

Definition 2.5. [7] Let $(H, \circ)$ be a hypergroup (or $H_{v}$-group) and $A$ be a fuzzy subset of $H$ with membership function $\mu_{A}(x) \in[0,1]$. Then $A$ is a fuzzy subhypergroup (or $H_{v}$-subgroup) of $H$ if the following conditions hold:

(1) $\inf \left\{\mu_{A}(z): z \in x \circ y\right\} \geq \min \left\{\mu_{A}(x), \mu_{A}(y)\right\}$ for all $x, y \in H$;

(2) For all $x, a \in H$, there exists $y \in H$ such that $x \in a \circ y$ and $\min \left\{\mu_{A}(x), \mu_{A}(a)\right\} \leq \mu_{A}(y)$;

(3) For all $x, a \in H$, there exists $z \in H$ such that $x \in z \circ$ a and $\min \left\{\mu_{A}(x), \mu_{A}(a)\right\} \leq \mu_{A}(z)$.

Definition 2.6. [7] Let $(H, \circ)$ be a hypergroup (or $H_{v}$-group) and $A$ be a fuzzy subset of $H$ with membership function $\mu_{A}(x)$. Then $A$ is an anti-fuzzy subhypergroup (or $H_{v}$-subgroup) of $H$ if the following conditions hold:

(1) $\sup \left\{\mu_{A}(z): z \in x \circ y\right\} \leq \max \left\{\mu_{A}(x), \mu_{A}(y)\right\}$ for all $x, y \in H$;

(2) For all $x, a \in H$, there exists $y \in H$ such that $x \in a \circ y$ and $\mu_{A}(y) \leq \max \left\{\mu_{A}(x), \mu_{A}(a)\right\}$;

(3) For all $x, a \in H$, there exists $z \in H$ such that $x \in z \circ a$ and $\mu_{A}(z) \leq \max \left\{\mu_{A}(x), \mu_{A}(a)\right\}$.

Theorem 2.1. [7] Let $(H, \circ)$ be a hypergroup (or $H_{v}$-group) and $\mu$ be a fuzzy subset of $H$. Then $\mu$ is a fuzzy subhypergroup (or $H_{v}$-subgroup) of $H$ if and only if its complement $\mu^{c}$ is an anti-fuzzy subhypergroup (or $H_{v}$-subgroup) of $H$. Here, $\mu^{c}(x)=1-\mu(x)$ for all $x \in H$.

\section{Generalized traditional FuZZy subhyperstructures}

Davvaz et al. (see [7]) introduced the concept of generalized traditional fuzzy $H_{v}$-subgroup. In this section, we present their results. And we introduce the concept generalized traditional anti-fuzzy $H_{v^{-}}$ subgroup. 
Notation 3.1. Let $(H, \circ)$ be a hypergroup (or $H_{v}$-group) and $A$ be a fuzzy subset of $H$ with membership function $\mu_{A}(x)$. We say that:

(1) $x_{t} \in \mu_{A}$ if $\mu_{A}(x) \geq t$;

(2) $x_{t} \in q \mu_{A}$ if $\mu_{A}(x)+t>1$;

(3) $x_{t} \in \vee q \mu_{A}$ if $x^{t} \in \mu_{A}$ or $x^{t} \in q \mu_{A}$. Otherwise, we say that $x^{t} \in \overline{\nabla q \mu_{A}}$.

Definition 3.1. [7] Let $(H, \circ)$ be a hypergroup (or $H_{v}$-group) and $A$ be a fuzzy subset of $H$ with membership function $\mu_{A}(x)$. Then $A$ is an $(\in, \in \vee q)$ fuzzy subhypergroup (or $H_{v}$-subgroup) of $H$ if for all $\left.\left.t, s \in\right] 0,1\right]$ and $x, y \in H$, the following conditions hold:

(1) $x_{t}, y_{s} \in \mu$ implies $z_{t \wedge s} \in \vee q \mu$ for all $z \in x \circ y$;

(2) $x_{t}, a_{s} \in \mu$ implies $y_{t \wedge s} \in \vee q \mu$ for some $y \in H$ such that $x \in a \circ y$;

(3) $x_{t}, a_{s} \in \mu$ implies $y_{t \wedge s} \in \vee q \mu$ for some $y \in H$ such that $x \in y \circ a$.

Theorem 3.1. [7] Let $(H, \circ)$ be a hypergroup (or $H_{v}$-group) and $A$ be a fuzzy subset of $H$ with membership function $\mu_{A}(x)$. Then $A$ is an $(\in, \in \vee q)$ fuzzy subhypergroup (or $H_{v}$-subgroup) of $H$ if and only if for all $x, y \in H$, the following conditions hold:

(1) $\mu_{A}(x) \wedge \mu_{A}(y) \wedge 0.5 \leq \mu_{A}(z)$ for all $z \in x \circ y$;

(2) For all $x, a \in H$, there exists $y \in H$ such that $x \in a \circ y$ and $\mu_{A}(x) \wedge \mu_{A}(a) \wedge 0.5 \leq \mu_{A}(y)$;

(3) For all $x, a \in H$, there exists $y \in H$ such that $x \in y \circ a$ and $\mu_{A}(x) \wedge \mu_{A}(a) \wedge 0.5 \leq \mu_{A}(y)$.

Definition 3.2. Let $A=\left\{\left(x, \mu_{A}(x)\right): x \in U\right\}$ be a fuzzy set. Then the set $A_{\pi}=\left\{\left(x, 2 \pi \mu_{A}(x)\right): x \in U\right\}$ is said to be a $\pi$-fuzzy set.

Proposition 3.1. Let $(H, \circ)$ be a hypergroup (or $H_{v}$-group). A $\pi$-fuzzy set $A_{\pi}$ is an $(\in, \in \vee q) \pi$-fuzzy subhypergroup (or $H_{v}$-subgroup) of $H$ if and only if $A$ is an $(\in, \in \vee q)$ fuzzy subhypergroup (or $H_{v}$-subgroup) of $H$.

Proof. The proof is straightforward.

Notation 3.2. Let $(H, \circ)$ be a hypergroup (or $H_{v}$-group) and $A$ be a fuzzy subset of $H$ with membership function $\mu_{A}(x)$. We say that:

(1) $x^{t} \in \mu_{A}$ if $\mu_{A}(x) \leq t$;

(2) $x^{t} \in \widehat{q} \mu_{A}$ if $\mu_{A}(x)+t<1$;

(3) $x^{t} \in \vee \widehat{q} \mu_{A}$ if $x^{t} \in \mu_{A}$ or $x^{t} \in \widehat{q} \mu_{A}$. Otherwise, we say that $x^{t} \in \bar{\vee} \widehat{q} \mu_{A}$.

Definition 3.3. Let $(H, \circ)$ be a hypergroup (or $H_{v}$-group) and $A$ be a fuzzy subset of $H$ with membership function $\mu_{A}(x)$. Then $A$ is an $\left(\in, \in \vee \widehat{q}\right.$ ) anti-fuzzy subhypergroup (or $H_{v}$-subgroup) of $H$ if for all $\left.\left.t, s \in\right] 0,1\right]$ and $x, y \in H$, the following conditions hold: 
(1) $x^{t}, y^{s} \in \mu$ implies $z^{t \vee s} \in \vee \widehat{q} \mu$ for all $z \in x \circ y$;

(2) $x^{t}, a^{s} \in \mu$ implies $y^{t \vee s} \in \vee \widehat{q} \mu$ for some $y \in H$ such that $x \in a \circ y$;

(3) $x^{t}, a^{s} \in \mu$ implies $y^{t \vee s} \in \vee \widehat{q} \mu$ for some $y \in H$ such that $x \in y \circ a$.

Theorem 3.2. Let $(H, \circ)$ be a hypergroup (or $H_{v}$-group) and $A$ be a fuzzy subset of $H$ with membership function $\mu_{A}(x)$. Then $A$ is an $\left(\in, \in \vee \widehat{q}\right.$ ) anti-fuzzy subhypergroup (or $H_{v}$-subgroup) of $H$ if and only if for all $x, y \in H$, the following conditions hold:

$\left(1^{\prime}\right) \mu_{A}(x) \vee \mu_{A}(y) \vee 0.5 \geq \vee \mu_{A}(z)$ for all $z \in x \circ y$,

$\left(2^{\prime}\right)$ For all $x, a \in H$, there exists $y \in H$ such that $x \in a \circ y$ and $\mu_{A}(x) \vee \mu_{A}(a) \vee 0.5 \geq \mu_{A}(y)$,

$\left(3^{\prime}\right)$ For all $x, a \in H$, there exists $y \in H$ such that $x \in y \circ a$ and $\mu_{A}(x) \vee \mu_{A}(a) \vee 0.5 \geq \mu_{A}(y)$.

Proof. $\left(1 \Rightarrow 1^{\prime}\right)$ : Suppose that $x, y \in H$. We consider the following cases:

- Case $\mu(x) \vee \mu(y)<0.5$. Assume, to get contradiction, that there exists $z \in x \circ y$ such that $\mu(z)>0.5$. It is clear that $x^{0.5}, y^{0.5} \in \mu$ and that $z^{0.5}$ is not in $\mu$. Having that $\mu(z)+0.5>1$ implies that $z^{0.5}$ is not in $\widehat{q} \mu$. We get that $z^{0.5} \in \bar{\nabla} \widehat{q} \mu$.

- Case $\mu(x) \vee \mu(y) \geq 0.5$. Assume, to get contradiction, that there exists $z \in x \circ y$ such that $\mu(z)>$ $\mu(x) \vee \mu(y) \vee 0.5 \geq 0.5$. Choose a real number $t$ such that $0.5 \leq \mu(x) \vee \mu(y)<t<\mu(z)$. It is clear that $x^{t}, y^{t} \in \mu$ and that $z^{t}$ is not in $\mu$. Having that $\mu(z)+t>1$ implies that $z^{t}$ is not in $\widehat{q} \mu$. We get that $z^{t} \in \bar{\vee} \widehat{q} \mu$.

$\left(2 \Rightarrow 2^{\prime}\right)$ : Suppose that $x, a \in H$. We consider the following cases:

- Case $\mu(x) \vee \mu(a)<0.5$. Assume, to get contradiction, that for every $y \in H$ such that $x \in a \circ y$ we have $\mu(y)>\mu(a) \vee \mu(x) \vee 0.5=0.5$. It is clear that $x^{0.5}, a^{0.5} \in \mu$ and that $y^{0.5}$ is not in $\mu$. Having that $\mu(y)+0.5>1$ implies that $y^{0.5}$ is not in $\widehat{q} \mu$. We get that $y^{0.5} \in \bar{\nabla} \widehat{q} \mu$.

- Case $\mu(x) \vee \mu(a) \geq 0.5$. Assume, to get contradiction, that for every $y \in H$ such that $x \in a \circ y$ we have $\mu(y)>\mu(a) \vee \mu(x) \vee 0.5=\mu(a) \vee \mu(x)$. Choose a real number $t$ such that $0.5 \leq \mu(x) \vee \mu(a)<t<\mu(y)$. It is clear that $x^{t}, a^{t} \in \mu$ and that $y^{t}$ is not in $\mu$. Having that $\mu(y)+t>1$ implies that $y^{t}$ is not in $\widehat{q} \mu$. We get that $y^{t} \in \overline{\mathrm{V} \widehat{q} \mu}$.

$\left(3 \Rightarrow 3^{\prime}\right)$ : Can be done in a similar manner to $\left(2 \Rightarrow 2^{\prime}\right)$.

$\left(1^{\prime} \Rightarrow 1\right)$ : Let $x^{t}, y^{s} \in \mu$. Then $\mu(x) \leq t, \mu(y) \leq t$. Let $z \in x \circ y$. We consider the following cases:

- Case $t \vee s<0.5$. We obtain that $\mu(z) \leq \mu(x) \vee \mu(y) \vee 0.5 \leq t \vee s \vee 0.5 \leq 0.5$. Then $\mu(z)+t \vee s<1$. Then $z^{t \vee s} \in \widehat{q} \mu$.

- Case $t \vee s \geq 0.5$. We obtain that $\mu(z) \leq \mu(x) \vee \mu(y) \vee 0.5 \leq t \vee s \vee 0.5 \leq t \vee s$. Then $z^{t \vee s} \in \mu$.

$\left(2^{\prime} \Rightarrow 2\right)$ : Let $x^{t}, a^{s} \in \mu$. Then $\mu(x) \leq t, \mu(y) \leq t$. Let $y \in H$ such that $x \in a \circ y$. We consider the following cases: 
- Case $t \vee s<0.5$. We get that $\mu(y) \leq \mu(x) \vee \mu(a) \vee 0.5 \leq t \vee s \vee 0.5 \leq 0.5$. Then $\mu(y)+t \vee s \in \mu<1$.

- Case $t \vee s \geq 0.5$. We get that $\mu(y) \leq \mu(x) \vee \mu(a) \vee 0.5 \leq t \vee s \vee 0.5 \leq t \vee s$. Then $y^{t \vee s} \in \mu$.

$\left(3^{\prime} \Rightarrow 3\right)$ : Can be done in a similar manner to $\left(2^{\prime} \Rightarrow 2\right)$.

Proposition 3.2. Let $(H, \circ)$ be a hypergroup (or $H_{v}$-group). A $\pi$-fuzzy set $A_{\pi}$ is an $(\in, \in \vee \widehat{q}) \pi$-antifuzzy subhypergroup (or $H_{v}$-subgroup) of $H$ if and only if $A$ is an $(\in, \in \vee \widehat{q}$ ) anti-fuzzy subhypergroup (or $H_{v}$-subgroup) of $H$.

Proof. The proof is straightforward.

Theorem 3.3. Let $(H, \circ)$ be a hypergroup (or $H_{v}$-group) and $A$ be a fuzzy subset of $H$ with membership function $\mu_{A}(x)$. Then $A$ is an $(\in, \in \vee q)$ fuzzy subhypergroup (or $H_{v}$-subgroup) of $H$ if and only if its complement, $A^{c}$, is an $\left(\in, \in \vee \widehat{q}\right.$ ) anti-fuzzy subhypergroup (or $H_{v}$-subgroup) of $H$.

Proof. Let $\mu_{A}$ be an $(\in, \in \vee q)$ fuzzy subhypergroup (or $H_{v}$-subgroup). Then the conditions of Theorem 3.1 are satisfied and we need to show that the conditions of Theorem 3.2 are satisfied. To prove $\left(1^{\prime}\right)$, let $x, y \in H$. For all $z \in x \circ y$, we have that $\mu_{A}(x) \wedge \mu_{A}(y) \wedge 0.5 \leq \mu_{A}(z)$. We get now that $1-\min \left\{\mu_{A}(x), \mu_{A}(y), 0.5\right\} \geq$ $1-\mu_{A}(z)$. The latter implies that $\max \left\{\mu_{A}^{c}(x), \mu_{A}^{c}(y), 0.5\right\} \geq \mu_{A}^{c}(z)$.

To prove $\left(2^{\prime}\right)$, let $x, a \in H$. Then there exists $y \in H$ such that $x \in a \circ y$ and $\mu_{A}(x) \wedge \mu_{A}(a) \wedge 0.5 \leq \mu_{A}(y)$. We get now that $1-\min \left\{\mu_{A}(x), \mu_{A}(a), 0.5\right\} \geq 1-\mu_{A}(y)$. The latter implies that $\max \left\{\mu_{A}^{c}(x), \mu_{A}^{c}(a), 0.5\right\} \geq \mu_{A}^{c}(y)$. In a similar manner, we can prove the validity of condition $\left(3^{\prime}\right)$. Thus, $\mu_{A}^{c}$, is an $(\in, \in \vee q)$ anti-fuzzy subhypergroup (or $H_{v}$-subgroup) of $H$.

Let $\mu_{A}^{c}$ be an $\left(\in, \in \vee \widehat{q}\right.$ ) anti-fuzzy subhypergroup (or $H_{v}$-subgroup). Then the conditions of Theorem 3.2 are satisfied and we need to show that the conditions of Theorem 3.1 are satisfied.. To prove (1), let $x, y \in H$. For all $z \in x \circ y$, we have that $\mu_{A}^{c}(x) \vee \mu_{A}^{c}(y) \vee 0.5 \geq \mu_{A}^{c}(z)$. We get now that $1-\max \left\{\mu_{A}^{c}(x), \mu_{A}^{c}(y), 0.5\right\} \leq$ $1-\mu_{A}^{c}(z)$. The latter implies that $\min \left\{\mu_{A}(x), \mu_{A}(y), 0.5\right\} \leq \mu_{A}(z)$.

In order to prove (2), let $x, a \in H$. Then there exists s $y \in H$ such that $x \in a \circ y$ and $\mu_{A}^{c}(x) \vee$ $\mu_{A}^{c}(a) \vee 0.5 \geq \mu_{A}^{c}(y)$. We get now that $1-\max \left\{\mu_{A}^{c}(x), \mu_{A}^{c}(a), 0.5\right\} \leq 1-\mu_{A}^{c}(y)$. The latter implies that $\min \left\{\mu_{A}(x), \mu_{A}(a), 0.5\right\} \leq \mu_{A}(y)$. In a similar manner, we can prove the validity of condition (3). Thus, $\mu_{A}$, is an $(\in, \in \vee q)$ fuzzy subhypergroup (or $H_{v}$-subgroup) of $H$.

\section{Generalized COMPLEX FUZZY AND ANTI-FUZZY SUBHYPERSTRUCTURES}

In this section, we use the concept of generalized fuzzy subhypergroups, discussed in Section 3 , to define complex fuzzy (anti-fuzzy) subhypergroups. And we investigate their properties.

\subsection{Generalized complex fuzzy $H_{v}$-subgroups.}


Definition 4.1. [10] A complex fuzzy set, defined on a universe of discourse $U$ is characterized by a membership function $\mu_{A}(x)$ that assigns any element a complex-valued grade of membership in $A$. The complex fuzzy set may be represented by the set of ordered pairs

$$
A=\left\{\left(x, \mu_{A}(x)\right): x \in U\right\}
$$

where $\mu_{A}(x)=r(x) e^{i w(x)}, i=\sqrt{-1}, r(x) \in[0,1]$ and $w(x) \in[0,2 \pi]$.

Remark 4.1. By setting $w(x)=0$ in the above definition, we return back to the traditional fuzzy set.

Definition 4.2. Let $A=\left\{\left(x, \mu_{A}(x)\right): x \in H\right\}$ be complex fuzzy subset of a non-void set $H$ with membership function $\mu_{A}(x)=r_{A}(x) e^{i w_{A}(x)}$. Then $A$ is said to be homogeneous if for all $x, y \in H$, we have

$$
r_{A}(x) \leq r_{A}(y) \text { if and only if } w_{A}(x) \leq w_{A}(y)
$$

Notation 4.1. Let $A=\left\{\left(x, \mu_{A}(x)\right): x \in H\right\}$ and $B=\left\{\left(x, \mu_{B}(x)\right): x \in H\right\}$ be complex fuzzy subsets of a non-void set $H$ with membership functions $\mu_{A}(x)=r_{A}(x) e^{i w_{A}(x)}$ and $\mu_{B}(x)=r_{B}(x) e^{i w_{B}}(x)$ respectively. By $\mu_{A}(x) \leq \mu_{B}(x)$, we mean that $r_{A}(x) \leq r_{B}(x)$ and $w_{A}(x) \leq w_{B}(x)$.

Throughout this paper, all complex fuzzy sets are considered homogeneous.

Notation 4.2. Let $(H, \circ)$ be a hypergroup (or $H_{v}$-group) and $A$ be a complex fuzzy subset of $H$ with membership function $\mu_{A}(x)=r_{A}(x) e^{i w_{A}(x)}$. We say, for all $0 e^{0 i}<t=s e^{i \theta} \leq 1 e^{2 \pi i}$, that:

(1) $x_{t} \in \mu_{A}$ if $r_{A}(x) \geq s$ and $w_{A}(x) \geq \theta$;

(2) $x_{t} \in q \mu_{A}$ if $r_{A}(x)+s>1$ and $w_{A}(x)+\theta>2 \pi$;

(3) $x_{t} \in \vee q \mu_{A}$ if $x^{t} \in \mu_{A}$ or $x^{t} \in q \mu_{A}$. Otherwise, we say that $x^{t} \in \overline{\nabla q \mu_{A}}$.

Definition 4.3. Let $(H, \circ)$ be a hypergroup (or $H_{v}$-group) and $A$ be a (homogeneous) complex fuzzy subset of $H$ with membership function $\mu_{A}(x)=r_{A}(x) e^{i w_{A}(x)}$. Then $A$ is an $(\in, \in \vee q)$ complex fuzzy subhypergroup (or $H_{v}$-subgroup) of $H$ if for all $\left.\left.t, s \in\right] 0 e^{0 i}, 1 e^{2 \pi i}\right]$ and $x, y \in H$, the following conditions hold:

(1) $x_{t}, y_{s} \in \mu$ implies $z_{t \wedge s} \in \vee q \mu$ for all $z \in x \circ y$;

(2) $x_{t}, a_{s} \in \mu$ implies $y_{t \wedge s} \in \vee q \mu$ for some $y \in H$ such that $x \in a \circ y$;

(3) $x_{t}, a_{s} \in \mu$ implies $y_{t \wedge s} \in \vee q \mu$ for some $y \in H$ such that $x \in y \circ a$.

Theorem 4.1. Let $(H, \circ)$ be a hypergroup (or $H_{v}$-group) and $A$ be a (homogeneous) complex fuzzy subset of $H$ with membership function $\mu_{A}(x)$. Then $A$ is an $(\in, \in \vee q)$ complex fuzzy subhypergroup (or $H_{v}$-subgroup) of $H$ if and only if for all $x, y \in H$, the following conditions hold:

$\left(1^{\prime}\right): \mu_{A}(x) \wedge \mu_{A}(y) \wedge 0.5 e^{i \pi} \leq \mu_{A}(z)$ for all $z \in x \circ y$,

$\left(2^{\prime}\right):$ For all $x, a \in H$, there exists $y \in H$ such that $x \in a \circ y$ and $\mu_{A}(x) \wedge \mu_{A}(a) \wedge 0.5 e^{i \pi} \leq \mu_{A}(y)$, 
$\left(3^{\prime}\right):$ For all $x, a \in H$, there exists $y \in H$ such that $x \in y \circ a$ and $\mu_{A}(x) \wedge \mu_{A}(a) \wedge 0.5 e^{i \pi} \leq \mu_{A}(y)$.

Proof. $\left(1 \Rightarrow 1^{\prime}\right)$ : Suppose that $x, y \in H$. We consider the following cases:

- Case $\mu(x) \wedge \mu(y)<0.5 e^{i \pi}$. Assume, to get contradiction, that there exists $z \in x \circ y$ such that $\mu(z)<\mu(x) \wedge \mu(y) \wedge 0.5 e^{i \pi} \leq 0.5 e^{i \pi}$. Choose a real number $t$ such that $\mu(z)<t \leq \mu(x) \wedge \mu(y)<0.5 e^{i \pi}$. It is clear that $x_{t}, y_{t} \in \mu$ and that $z_{t}$ is not in $\mu$. Having that $\mu(z)+t<1 e^{2 i \pi}$ implies that $z_{t}$ is not in $q \mu$. We get that $z_{t} \in \overline{\nabla q \mu}$.

- Case $\mu(x) \wedge \mu(y) \geq 0.5 e^{i \pi}$. Assume, to get contradiction, that there exists $z \in x \circ y$ such that $\mu(z)<$ $0.5 e^{i \pi}$. It is clear that $x_{0.5 e^{i \pi}}, y_{0.5 e^{i \pi}} \in \mu$ and that $z_{0.5 e^{i \pi}}$ is not in $\mu$. Having that $\mu(z)+0.5 e^{i \pi}<1 e^{2 i \pi}$ implies that $z_{0.5 e^{i \pi}}$ is not in $q \mu$. We get that $z_{0.5 e^{i \pi}} \in \overline{\nabla q \mu}$.

$\left(2 \Rightarrow 2^{\prime}\right)$ : Suppose that $x, a \in H$. We consider the following cases:

- Case $\mu(x) \wedge \mu(a) \leq 0.5 e^{i \pi}$. Assume, to get contradiction, that for every $y \in H$ such that $x \in a \circ y$ we have $\mu(y)<\mu(a) \wedge \mu(x) \wedge 0.5 e^{i \pi}=\mu(a) \wedge \mu(x)$. Choose a real number $t$ such that $\mu(y)<t<$ $\mu(x) \wedge \mu(a)<0.5 e^{i \pi}$. It is clear that $x_{t}, a_{t} \in \mu$ and that $y_{t}$ is not in $\mu$. Having that $\mu(y)+t<1 e^{2 i \pi}$ implies that $y_{t}$ is not in $q \mu$. We get that $y_{t} \in \overline{\nabla q \mu}$.

- Case $\mu(x) \wedge \mu(a)>0.5 e^{i \pi}$. Assume, to get contradiction, that for every $y \in H$ such that $x \in a \circ y$ we

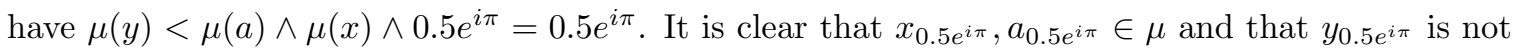
in $\mu$. Having that $\mu(y)+0.5 e^{i \pi}<1 e^{2 i \pi}$ implies that $y_{0.5 e^{i \pi}}$ is not in $q \mu$. We get that $y_{0.5 e^{i \pi} \in \bar{V} q \mu}$. $\left(3 \Rightarrow 3^{\prime}\right)$ : Can be done in a similar manner to $\left(2 \Rightarrow 2^{\prime}\right)$.

$\left(1^{\prime} \Rightarrow 1\right):$ Let $x_{t}, y_{s} \in \mu$. Then $\mu(x) \leq t, \mu(y) \leq s$. Let $z \in x \circ y$. We consider the following cases:

- Case $t \wedge s \leq 0.5 e^{i \pi}$. We get that $\mu(z) \geq \mu(x) \wedge \mu(y) \wedge 0.5 e^{i \pi} \geq t \wedge s \wedge 0.5 e^{i \pi} \geq t \wedge s$. Then $z_{t \wedge s} \in \mu$.

- Case $t \wedge s>0.5 e^{i \pi}$. We get that $\mu(z) \geq \mu(x) \wedge \mu(y) \wedge 0.5 e^{i \pi} \geq t \wedge s \wedge 0.5 e^{i \pi} \geq 0.5 e^{i \pi}$. Then $\mu(z)+t \wedge s>1 e^{2 i \pi}$. Thus, $z_{t \wedge s} \in \widehat{q} \mu$.

$\left(2^{\prime} \Rightarrow 2\right)$ : Let $x_{t}, a_{s} \in \mu$. Then $\mu(x) \leq t, \mu(y) \leq t$. Let $y \in H$ such that $x \in a \circ y$. We consider the following cases:

- Case $t \wedge s \leq 0.5 e^{i \pi}$. We get that $\mu(y) \geq \mu(x) \wedge \mu(a) \wedge 0.5 e^{i \pi} \geq t \wedge s \wedge 0.5 e^{i \pi} \geq t \wedge s$. Then $y_{t \wedge s} \in \mu$.

- Case $t \wedge s>0.5 e^{i \pi}$. We get that $\mu(y) \geq \mu(x) \wedge \mu(a) \wedge 0.5 e^{i \pi} \geq t \wedge s \wedge 0.5 e^{i \pi} \geq 0.5 e^{i \pi}$. Then $\mu(y)+t \wedge s \in \mu>1 e^{2 i \pi}$.

$\left(3^{\prime} \Rightarrow 3\right)$ : Can be done in a similar manner to $\left(2^{\prime} \Rightarrow 2\right)$.

Theorem 4.2. Let $(H, \circ)$ be a hypergroup (or $H_{v}$-group) and $A$ be a (homogeneous) complex fuzzy subset of $H$ with membership function $\mu_{A}(x)=r_{A}(x) e^{i w_{A}(x)}$. Then $A$ is an $(\in, \in \vee q)$ complex fuzzy subhypergroup (or $H_{v}$-subgroup) of $H$ if and only if $r_{A}$ is an $(\in, \in \vee q)$ fuzzy subhypergroup (or $H_{v}$-subgroup) of $H$ and $w_{A}$ is an $(\in, \in \vee q) \pi$-fuzzy subhypergroup (or $H_{v}$-subgroup) of $H$. 
Proof. Let $A$ be an $(\in, \in \vee q)$ complex fuzzy subhypergroup (or $H_{v}$-subgroup) of $H$. This is equivalent to having the conditions of Theorem 4.1 satisfied and we can rewrite them as follows:

(1) $r_{A}(x) \wedge r_{A}(y) \wedge 0.5 \leq r_{A}(z)$ and $w_{A}(x) \wedge w_{A}(y) \wedge \pi \leq w_{A}(z)$ for all $z \in x \circ y$;

(2) For all $x, a \in H$, there exists $y \in H$ such that $x \in a \circ y$ and $r_{A}(x) \wedge r_{A}(a) \wedge 0.5 \leq r_{A}(y)$ and $w_{A}(x) \wedge w_{A}(a) \wedge \pi \leq w_{A}(y) ;$

(3) For all $x, a \in H$, there exists $y \in H$ such that $r_{A}(x) \wedge r_{A}(a) \wedge 0.5 \leq r_{A}(y)$ and $w_{A}(x) \wedge w_{A}(a) \wedge \pi \leq$ $w_{A}(y)$.

The latter conditions are equivalent to having $r_{A}$ an $(\in, \in \vee q)$ fuzzy subhypergroup (or $H_{v}$-subgroup) of $H$ and $w_{A}$ an $(\in, \in \vee q) \pi$-fuzzy subhypergroup (or $H_{v}$-subgroup) of $H$ as the conditions of Theorem 2.6 are satisfied for both: $r_{A}$ and $w_{A}$.

Proposition 4.1. Let $(H, \circ)$ be a hypergroup (or $H_{v}$-group) and $A$ be a (homogeneous) complex fuzzy subset of $H$ with membership function $\mu_{A}(x)=r_{A}(x) e^{i w_{A}(x)}$. If $A$ is a complex fuzzy subhypergroup (or $H_{v}$-subgroup) of $H$ then $A$ is an $(\in, \in \vee q)$ complex fuzzy subhypergroup (or $H_{v}$-subgroup) of $H$.

Proof. Let $A$ be a complex fuzzy subhypergroup (or $H_{v}$-subgroup) of $H$. Then the following conditions are satisfied for all $x, y \in H$ :

(1) $\mu_{A}(x) \wedge \mu_{A}(y) \leq \mu_{A}(z)$ for all $z \in x \circ y$;

(2) For all $x, a \in H$, there exists $y \in H$ such that $x \in a \circ y$ and $\mu_{A}(x) \wedge \mu_{A}(a) \leq \mu_{A}(y)$;

(3) For all $x, a \in H$, there exists $y \in H$ such that $x \in y \circ a$ and $\mu_{A}(x) \wedge \mu_{A}(a) \leq \mu_{A}(y)$.

We have that:

(1) $\mu_{A}(x) \wedge \mu_{A}(y) \wedge 0.5 e^{i \pi} \leq \mu_{A}(x) \wedge \mu_{A}(y) \leq \mu_{A}(z)$ for all $z \in x \circ y$,

(2) For all $x, a \in H$, there exists $y \in H$ such that $x \in a \circ y$ and $\mu_{A}(x) \wedge \mu_{A}(a) \wedge 0.5 e^{i \pi} \leq \mu_{A}(x) \wedge \mu_{A}(a) \leq$ $\mu_{A}(y)$,

(3) For all $x, a \in H$, there exists $y \in H$ such that $x \in y \circ a$ and $\mu_{A}(x) \wedge \mu_{A}(a) \wedge 0.5 e^{i \pi} \leq \mu_{A}(x) \wedge \mu_{A}(a) \leq$ $\mu_{A}(y)$.

Therefore, $A$ is an $(\in, \in \vee q)$ complex fuzzy subhypergroup (or $H_{v}$-subgroup) of $H$.

Remark 4.2. The converse of Proposition 4.1 is not always true. i.e., if $A$ is an $(\in, \in \vee q)$ complex fuzzy subhypergroup (or $H_{v}$-subgroup) of $H$ then $A$ may not be a complex fuzzy subhypergroup (or $H_{v}$-subgroup) of $H$.

We illustrate Remark 4.2 by the following example. 
Example 4.1. Let $H=\{0,1,2\}$ and define the $H_{v}$-group $(H,+)$ by the following table:

\begin{tabular}{|c|c|c|c|}
\hline+ & 0 & 1 & 2 \\
\hline 0 & 0 & $\{1,2\}$ & 2 \\
\hline 1 & $\{1,2\}$ & 2 & 0 \\
\hline 2 & 2 & 0 & 1 \\
\hline
\end{tabular}

And define a complex fuzzy subset $\mu$ of $H$ as: $\mu(0)=0.8 e^{i 2 \pi}, \mu(1)=0.7 e^{i \frac{3 \pi}{2}}$ and $\mu(2)=0.6 e^{i \pi}$. Then $\mu$ is an $(\in, \in \vee q)$ complex fuzzy $H_{v}$-subgroup of $H$ but it is not a complex fuzzy $H_{v}$-subgroup of $H$.

Theorem 4.3. Let $(H, \circ)$ be a hypergroup (or $H_{v}$-group) and $A$ be a (homogeneous) complex fuzzy subset of $H$ with membership function $\mu_{A}(x)$. Then $A$ is an $(\in, \in \vee q)$ complex fuzzy subhypergroup (or $H_{v}$-subgroup) of $H$ if and only if for all $0 e^{0 i}<t \leq 0.5 e^{\pi i}, \mu_{t}=\left\{x \in H: \mu_{A}(x) \geq t\right\} \neq \emptyset$ is a subhypergroup (or $H_{v}$-subgroup) of $H$.

Proof. The proof is similar to that in [7].

Proposition 4.2. Let $(H, \circ)$ be the biset hypergroup, i.e., $x \circ y=\{x, y\}$ for all $x, y \in H$ and let $\mu$ be any homogeneous complex fuzzy subset of $H$. Then $\mu$ is an $(\in, \in \vee q)$ complex fuzzy subhypergroup of $H$.

Proof. The proof follows from Proposition 4.1 and having $\mu$ a complex fuzzy subhypergroup of $H$ [1].

Proposition 4.3. Let $(H, \circ)$ be the total hypergroup, i.e., $x \circ y=H$ for all $x, y \in H$ and let $\mu$ be any homogeneous complex fuzzy subset of $H$. Then $\mu$ is an $(\in, \in \vee q)$ complex fuzzy subhypergroup of $H$ if and only if $\mu$ is a constant complex function or $0.5 e^{i \pi} \leq \mu(x) \leq 1 e^{2 i \pi}$ for all $x \in H$.

Proof. It is easy to see that if if $\mu$ is a constant complex function or $0.5 e^{i \pi} \leq \mu(x) \leq 1 e^{2 i \pi}$ for all $x \in H$ then $\mu$ is an $(\in, \in \vee q)$ complex fuzzy subhypergroup of $H$.

Let $\mu$ be an $(\in, \in \vee q)$ complex fuzzy subhypergroup of $H$ such that $\mu$ is not a constant complex function. Suppose, to get contradiction, that there exists $x \in H$ such that $\mu(x)=t<0.5 e^{i \pi}$. Then, by Theorem 4.3, $\mu_{t}=\left\{x \in H: \mu_{A}(x) \geq t\right\} \neq \emptyset$ is a subhypergroup (or $H_{v}$-subgroup) of $H$. The latter implies that for all $z \in H=x \circ x \mu(z) \geq t$. Since $\mu$ is not a constant function, it follows that we can find $y \in H$ such that $\mu(y)=t_{0} \neq t=\mu(x)$. We have two cases: $t_{0}<t$ and $t_{0}>t$. We consider the case $t_{0}<t$ and the other case can be done in a similar manner. Having $\mu_{t}$ a subhypergroup (or $H_{v}$-subgroup) of $H$ implies that $y \in H=x \circ x \subseteq \mu_{t}$.

Definition 4.4. Let $0 e^{0 i} \leq \alpha<\beta \leq 1 e^{2 \pi i}$ and $\mu$ be a (homogeneous) complex fuzzy subset of a hypergroup (or $H_{v}$-group) $H$. Then $\mu$ is called a complex fuzzy subhypergroup (or $H_{v}$-subgroup) with thresholds ( $\alpha, \beta$ ) of $H$ if for all $x, y \in H$, the following conditions are satisfied: 
(1) $\mu_{A}(x) \wedge \mu_{A}(y) \wedge \beta \leq \mu_{A}(z) \vee \alpha$ for all $z \in x \circ y$

(2) For all $x, a \in H$, there exists $y \in H$ such that $x \in a \circ y$ and $\mu_{A}(x) \wedge \mu_{A}(a) \wedge \beta \leq \mu_{A}(y) \vee \alpha$;

(3) For all $x, a \in H$, there exists $y \in H$ such that $x \in y \circ a$ and $\mu_{A}(x) \wedge \mu_{A}(a) \wedge \beta \leq \mu_{A}(y) \vee \alpha$.

Remark 4.3. If $\alpha=0 e^{0 i}, \beta=1 e^{i 2 \pi}$, then we obtain the complex fuzzy subhypergroup (or $H_{v}$-subgroup). And if $\alpha=0 e^{0 i}, \beta=0.5 e^{i \pi}$, we have an $(\in, \in \vee q)$ complex fuzzy subhypergroup (or $H_{v}$-subgroup) of $H$.

Theorem 4.4. Let $0 e^{0 i} \leq \alpha<\beta \leq 1 e^{2 \pi i}$ and $\mu$ be a (homogeneous) complex fuzzy subset of a hypergroup (or $H_{v}$-group) $H$. Then $\mu$ is a complex fuzzy subhypergroup (or $H_{v}$-subgroup) with thresholds $(\alpha, \beta)$ of $H$ if and only if $\mu_{t} \neq \emptyset$ is a subhypergroup (or $H_{v}$-subgroup) of $H$ for all $\left.\left.t \in\right] \alpha, \beta\right]$.

Proof. The proof is similar to that in [7].

\subsection{Generalized complex anti-fuzzy $H_{v}$-subgroups.}

Notation 4.3. Let $(H, \circ)$ be a hypergroup (or $H_{v}$-group) and $A$ be a complex fuzzy subset of $H$ with membership function $\mu_{A}(x)=r_{A}(x) e^{i w_{A}(x)}$. We say, for all $0 e^{0 i} \leq t=s e^{i \theta}<1 e^{2 \pi i}$, that:

(1) $x^{t} \in \mu_{A}$ if $r_{A}(x) \leq s$ and $w_{A}(x) \leq \theta$;

(2) $x^{t} \in \widehat{q} \mu_{A}$ if $r_{A}(x)+s<1$ and $w_{A}(x)+\theta<2 \pi$;

(3) $x^{t} \in \vee \widehat{q} \mu_{A}$ if $x^{t} \in \mu_{A}$ or $x^{t} \in q \mu_{A}$. Otherwise, we say that $x^{t} \in \overline{\nabla q \mu_{A}}$.

Definition 4.5. Let $(H, \circ)$ be a hypergroup (or $H_{v}$-group) and $A$ be a (homogeneous) complex fuzzy subset of $H$ with membership function $\mu_{A}(x)$. Then $A$ is an $(\in, \in \vee \widehat{q})$ complex anti-fuzzy subhypergroup (or $H_{v^{-}}$ subgroup) of $H$ if for all $t, s \in\left[0 e^{0 i}, 1 e^{2 \pi i}[\right.$ and $x, y \in H$, the following conditions hold:

(1) $x^{t}, y^{s} \in \mu$ implies $z^{t \vee s} \in \vee \widehat{q} \mu$ for all $z \in x \circ y$;

(2) $x^{t}, a^{s} \in \mu$ implies $y^{t \vee s} \in \vee \widehat{q} \mu$ for some $y \in H$ such that $x \in a \circ y$;

(3) $x^{t}, a^{s} \in \mu$ implies $y^{t \vee s} \in \vee \widehat{q} \mu$ for some $y \in H$ such that $x \in y \circ a$.

Theorem 4.5. Let $(H, \circ)$ be a hypergroup (or $H_{v}$-group) and $A$ be a fuzzy subset of $H$ with membership function $\mu_{A}(x)$. Then $A$ is an $\left(\in, \in \vee \widehat{q}\right.$ ) anti-fuzzy subhypergroup (or $H_{v}$-subgroup) of $H$ if and only if for all $x, y \in H$, the following conditions hold:

(1) $\mu_{A}(x) \vee \mu_{A}(y) \vee 0.5 e^{i \pi} \geq \mu_{A}(z)$ for all $\left.z \in x \circ y\right\}$;

(2) For all $x, a \in H$, there exists $y \in H$ such that $x \in a \circ y$ and $\mu_{A}(x) \vee \mu_{A}(a) \vee 0.5 e^{i \pi} \geq \mu_{A}(y)$;

(3) For all $x, a \in H$, there exists $y \in H$ such that $x \in y \circ a$ and $\mu_{A}(x) \vee \mu_{A}(a) \vee 0.5 e^{i \pi} \geq \mu_{A}(y)$.

Proof. The proof is similar to that of Theorem 3.2.

Theorem 4.6. Let $(H, \circ)$ be a hypergroup (or $H_{v}$-group) and $A$ be a (homogeneous) complex fuzzy subset of $H$ with membership function $\mu_{A}(x)=r_{A}(x) e^{i w_{A}(x)}$. Then $A$ is an $(\in, \in \vee \widehat{q})$ complex anti-fuzzy subhypergroup 
(or $H_{v}$-subgroup) of $H$ if and only if $r_{A}$ is an $\left(\in, \in \vee \widehat{q}\right.$ ) fuzzy subhypergroup (or $H_{v}$-subgroup) of $H$ and $w_{A}$ is an $\left(\in, \in \vee \widehat{q}\right.$ ) $\pi$-anti-fuzzy subhypergroup (or $H_{v}$-subgroup) of $H$.

Proof. Let $A$ be an $\left(\in, \in \vee \widehat{q}\right.$ ) complex fuzzy subhypergroup (or $H_{v}$-subgroup) of $H$. This is equivalent to having the conditions of Theorem 4.5 satisfied and we can rewrite them as follows:

(1) $r_{A}(x) \vee r_{A}(y) \vee 0.5 \geq r_{A}(z)$ and $w_{A}(x) \vee w_{A}(y) \vee \pi \geq w_{A}(z)$ for all $z \in x \circ y$;

(2) For all $x, a \in H$, there exists $y \in H$ such that $x \in a \circ y$ and $r_{A}(x) \vee r_{A}(a) \vee 0.5 \geq r_{A}(y)$ and $w_{A}(x) \vee w_{A}(a) \vee \pi \geq w_{A}(y) ;$

(3) For all $x, a \in H$, there exists $y \in H$ such that $r_{A}(x) \vee r_{A}(a) \vee 0.5 \geq r_{A}(y)$ and $w_{A}(x) \vee w_{A}(a) \vee \pi \geq$ $w_{A}(y)$.

The latter conditions are equivalent to having $r_{A}$ is an $(\in, \in \vee \widehat{q})$ anti-fuzzy subhypergroup (or $H_{v}$-subgroup) of $H$ and $w_{A}$ is an $(\in, \in \vee \widehat{q}) \pi$-anti-fuzzy subhypergroup (or $H_{v}$-subgroup) of $H$ as the conditions of Theorem 3.2 are satisfied for both: $r_{A}$ and $w_{A}$.

Theorem 4.7. Let $(H, \circ)$ be a hypergroup (or $H_{v}$-group) and $A$ be a (homogeneous) complex fuzzy subset of $H$ with membership function $\mu_{A}(x)$. Then $A$ is an $(\in, \in \vee q)$ complex fuzzy subhypergroup (or $H_{v}$-subgroup) of $H$ if and only if its complement, $A^{c}$, is an $(\in, \in \vee \widehat{q})$ complex anti-fuzzy subhypergroup (or $H_{v}$-subgroup) of $H$.

Proof. The proof results from Theorems 3.3, 4.2 and 4.6.

Proposition 4.4. Let $(H, \circ)$ be a hypergroup (or $H_{v}$-group) and $A$ be a (homogeneous) complex fuzzy subset of $H$ with membership function $\mu_{A}(x)=r_{A}(x) e^{i w_{A}(x)}$. If $A$ is a complex anti-fuzzy subhypergroup (or $H_{v}$-subgroup) of $H$ then $A$ is an $\left(\in, \in \vee \widehat{q}\right.$ ) complex anti-fuzzy subhypergroup (or $H_{v}$-subgroup) of $H$.

Proof. Let $A$ be a complex anti-fuzzy subhypergroup (or $H_{v}$-subgroup) of $H$. Then the following conditions are satisfied for all $x, y \in H$ :

(1) $\mu_{A}(x) \vee \mu_{A}(y) \geq \mu_{A}(z)$ for all $z \in x \circ y$;

(2) For all $x, a \in H$, there exists $y \in H$ such that $x \in a \circ y$ and $\mu_{A}(x) \vee \mu_{A}(a) \geq \mu_{A}(y)$;

(3) For all $x, a \in H$, there exists $y \in H$ such that $x \in y \circ a$ and $\mu_{A}(x) \vee \mu_{A}(a) \geq \mu_{A}(y)$.

We have that:

(1) $\mu_{A}(x) \vee \mu_{A}(y) \vee 0.5 e^{i \pi} \geq \mu_{A}(x) \vee \mu_{A}(y) \geq \mu_{A}(z)$ for all $z \in x \circ y$;

(2) For all $x, a \in H$, there exists $y \in H$ such that $x \in a \circ y$ and $\mu_{A}(x) \vee \mu_{A}(a) \vee 0.5 e^{i \pi} \geq \mu_{A}(x) \vee \mu_{A}(a) \geq$ $\mu_{A}(y)$

(3) For all $x, a \in H$, there exists $y \in H$ such that $x \in y \circ a$ and $\mu_{A}(x) \vee \mu_{A}(a) \vee 0.5 e^{i \pi} \geq \mu_{A}(x) \vee \mu_{A}(a) \geq$ $\mu_{A}(y)$. 
Therefore, $A$ is an $\left(\in, \in \vee \widehat{q}\right.$ ) complex anti-fuzzy subhypergroup (or $H_{v}$-subgroup) of $H$.

Remark 4.4. The converse of Proposition 4.1 is not always true, i.e., if $A$ is an $(\in, \in \vee \widehat{q})$ complex anti-fuzzy subhypergroup (or $H_{v}$-subgroup) of $H$ then $A$ may not be a complex anti-fuzzy subhypergroup (or $H_{v}$-subgroup) of $H$.

We illustrate Remark 4.4 by the following example.

Example 4.2. Let $H=\{0,1,2\}$ and define the $H_{v}$-group $(H,+)$ by the following table:

\begin{tabular}{|c|c|c|c|}
\hline+ & 0 & 1 & 2 \\
\hline 0 & 0 & $\{1,2\}$ & 2 \\
\hline 1 & $\{1,2\}$ & 2 & 0 \\
\hline 2 & 2 & 0 & 1 \\
\hline
\end{tabular}

And define a complex fuzzy subset $\mu$ of $H$ as: $\mu(0)=0.2 e^{i 0}, \mu(1)=0.3 e^{i \frac{\pi}{2}}$ and $\mu(2)=0.4 e^{i \pi}$. Then $\mu$ is an $(\in, \in \vee \widehat{q})$ complex anti-fuzzy $H_{v}$-subgroup of $H$ but it is not a complex anti-fuzzy $H_{v}$-subgroup of $H$.

Theorem 4.8. Let $(H, \circ)$ be a hypergroup (or $H_{v}$-group) and $A$ be a (homogeneous) complex fuzzy subset of $H$ with membership function $\mu_{A}(x)$. Then $A$ is an $\left(\in, \in \vee \widehat{q}\right.$ ) complex fuzzy subhypergroup (or $H_{v}$-subgroup) of $H$ if and only if for all $0.5 e^{\pi i} \leq t<1 e^{2 \pi i}, \mu^{t}=\left\{x \in H: \mu_{A}(x) \leq t\right\} \neq \emptyset$ is a subhypergroup (or $H_{v}$-subgroup) of $H$.

Proof. Let $\mu_{A}$ be an $(\in, \in \vee q)$ complex fuzzy subhypergroup (or $H_{v^{-}}$subgroup) of $H$ and let $0.5 e^{\pi i} \leq t<$ $1 e^{2 \pi i}$. We need to show that $a \circ \mu^{t}=\mu^{t} \circ a=\mu^{t}$ for all $a \in \mu^{t}$. We prove that $a \circ \mu^{t}=\mu^{t}$ and $\mu^{t} \circ a=\mu^{t}$ can be done in a similar manner. Let $x \in \mu^{t}$. Then $\mu_{A}(x) \leq t$ and $\mu_{A}(a) \leq t$. Since $\mu_{A}$ is an $(\in, \in \vee q)$ complex fuzzy

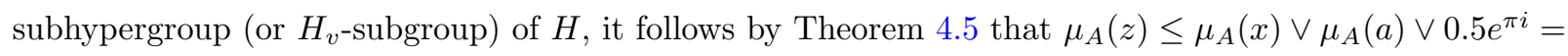
leqt $\vee 0.5 e^{\pi i} \leq t$ for all $z \in x \circ a$. The latter implies that $a \circ \mu^{t} \subseteq \mu^{t}$. To prove that $\mu^{t} \subseteq a \circ \mu^{t}$, let $x \in \mu^{t}$. Then, by Theorem 4.5, there exists $y \in H$ such that $x \in a \circ y$ and $\mu_{A}(y) \leq \mu_{A}(x) \vee \mu_{A}(a) \vee 0.5 e^{\pi i}=\leq t \vee 0.5 e^{\pi i} \leq t$. Thus, $y \in \mu^{t}$ and $a \circ \mu^{t} \subseteq \mu^{t}$.

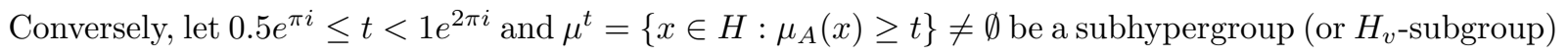
of $H$. Let $t_{0}=\mu_{A}(x) \vee \mu_{A}(y) \vee 0.5 e^{\pi i}$. Then $x, y \in \mu^{t_{0}}$. Since $\mu^{t_{0}}$ is a subhypergroup (or $H_{v}$-subgroup) of $H$, it follows that for all $z \in x \circ y$, we have $z \in \mu^{t_{0}}$. i.e., $\mu_{A}(x) \vee \mu_{A}(y) \vee 0.5 e^{\pi i}=t_{0} \geq \mu_{A}(z)$. Now let $x, a \in H$ and $t_{1}=\mu_{A}(x) \vee \mu_{A}(x) \vee 0.5 e^{\pi i}$. Then $x, a \in \mu^{t_{1}}$. Since $\mu^{t_{1}}$ is a subhypergroup (or $H_{v}$-subgroup) of $H$, it follows that there exists $y \in \mu^{t_{1}}$ such that $x \in a \circ y$. i.e., $\mu_{A}(x) \vee \mu_{A}(a) \vee 0.5 e^{\pi i}=t_{1} \geq \mu_{A}(y)$.

Proposition 4.5. Let $(H, \circ)$ be the biset hypergroup, i.e., $x \circ y=\{x, y\}$ for all $x, y \in H$ and let $\mu$ be any homogeneous complex fuzzy subset of $H$. Then $\mu$ is an $(\in, \in \vee \widehat{q})$ complex anti-fuzzy subhypergroup of $H$. 
Proof. The proof follows from Proposition 4.4 and having $\mu$ a complex anti-fuzzy subhypergroup of $H$ $([1])$.

Proposition 4.6. Let $(H, \circ)$ be the total hypergroup, i.e., $x \circ y=H$ for all $x, y \in H$ and let $\mu$ be any homogeneous complex fuzzy subset of $H$. Then $\mu$ is an $(\in, \in \vee \widehat{q})$ complex anti-fuzzy subhypergroup of $H$ if and only if $\mu$ is a constant complex function or $\mu(x) \leq 0.5 e^{i \pi}$ for all $x \in H$.

Proof. Let $\mu$ is an $(\in, \in \vee \widehat{q})$ complex anti-fuzzy subhypergroup of $H$. The statement is equivalent, by Theorem 4.7, we get that $\mu^{c}$ is an $(\in, \in \vee q)$ complex fuzzy subhypergroup of $H$. The latter is equivalent, using Proposition 4.3, to that $\mu^{c}$ is a constant complex function or $\mu^{c}(x) \geq 0.5 e^{i \pi}$ for all $x \in H$.

Definition 4.6. Let $0 e^{0 i} \leq \alpha<\beta \leq 1 e^{2 \pi i}$ and $\mu$ be a (homogeneous) complex fuzzy subset of a hypergroup (or $H_{v}$-group) $H$. Then $\mu$ is called a complex anti-fuzzy subhypergroup (or $H_{v}$-subgroup) with thresholds $(\alpha, \beta)$ of $H$ if for all $x, y \in H$, the following conditions are satisfied:

(1) $\mu_{A}(x) \vee \mu_{A}(y) \vee \alpha \geq \mu_{A}(z) \wedge \beta$ for all $z \in x \circ y$;

(2) For all $x, a \in H$, there exists $y \in H$ such that $x \in a \circ y$ and $\mu_{A}(x) \vee \mu_{A}(a) \vee \alpha \geq \mu_{A}(y) \wedge \beta$;

(3) For all $x, a \in H$, there exists $y \in H$ such that $x \in y \circ a$ and $\mu_{A}(x) \vee \mu_{A}(a) \vee \alpha \geq \mu_{A}(y) \wedge \beta$.

Remark 4.5. If $\alpha=0 e^{0 i}, \beta=1 e^{2 \pi i}$, we get the complex anti-fuzzy subhypergroup (or $H_{v}$-subgroup). And if $\alpha=0.5 e^{i \pi}, \beta=1 e^{i 2 \pi}$, we get an $(\in, \in \vee \widehat{q})$ complex fuzzy subhypergroup of $H$.

Theorem 4.9. Let $0 e^{0 i} \leq \alpha<\beta \leq 1 e^{2 \pi i}$ and $\mu$ be a (homogeneous) complex fuzzy subset of a hypergroup (or $H_{v}$-group) $H$. Then $\mu$ is a complex anti-fuzzy subhypergroup (or $H_{v}$-subgroup) with thresholds ( $\alpha, \beta$ ) of $H$ if and only if $\mu^{t} \neq \emptyset$ is a subhypergroup (or $H_{v}$-subgroup) of $H$ for all $t \in[\alpha, \beta[$.

Proof. Let $\mu$ is a complex fuzzy subhypergroup (or $H_{v}$-subgroup) with thresholds $(\alpha, \beta)$ of $H$. We need to show that $a \circ \mu^{t}=\mu^{t} \circ a=\mu^{t}$ for all $a \in \mu^{t}$. We prove that $a \circ \mu^{t}=\mu^{t}$ and $\mu^{t} \circ a=\mu^{t}$ can be done in a similar manner. Let $x \in \mu^{t}$. Then $\mu(x) \leq t$ and $\mu(a) \leq t$. Since $\mu$ is a complex anti-fuzzy subhypergroup (or $H_{v}$-subgroup) with thresholds $(\alpha, \beta)$ of $H$, it follows that $\mu(z) \wedge \beta \leq \mu(x) \vee \mu(a) \vee \alpha=t \vee \alpha \leq t$ for all $z \in x \circ a$. Since $\beta \geq t$, it follows that $\mu(z) \leq t$. The latter implies that $a \circ \mu^{t} \subseteq \mu^{t}$. To prove that $\mu^{t} \subseteq a \circ \mu^{t}$, let $x \in \mu^{t}$. Then, there exists $y \in H$ such that $x \in a \circ y$ and $\mu(y) \wedge \beta \leq \mu(x) \vee \mu(a) \vee \alpha=t \vee \alpha \leq t$. Thus, $y \in \mu^{t}$ and $a \circ \mu^{t} \subseteq \mu^{t}$.

Conversely, let $\alpha<t \leq \beta$ and $\mu^{t}=\{x \in H: \mu(x) \leq t\} \neq \emptyset$ be a subhypergroup (or $H_{v^{-}}$subgroup) of $H$. Suppose that there exists $z \in x \circ y$ such that $\mu(z) \wedge \beta>\mu(x) \vee \mu(y) \vee \alpha=t$. It is clear that $x, y \in \mu^{t}$ and $z$ is not in $\mu^{t}$ which contradicts our hypothesis that $\mu^{t}$ is a subhypergroup (or $H_{v}$-subgroup) of $H$. Thus, Condition 1. of Definition 4.6 is satisfied. Now assume that there exist $a, x \in H$ such that for all $y \in H, x \in a \circ y$, we have $\mu(y) \wedge \beta>\mu(x) \vee \mu(a) \vee \alpha=t_{0}$. It is clear that $x, a \in \mu^{t_{0}}$ and $y$ is not in $\mu^{t_{0}}$ 
which contradicts our hypothesis that $\mu^{t_{0}}$ is a subhypergroup (or $H_{v}$-subgroup) of $H$. Thus, Condition 2 . of Definition 4.6 is satisfied. We can prove Condition 3. in a similar manner.

\section{Conclusion}

This paper contributed to the study of fuzzy subhyperstructures by introducing the concepts of generalized complex fuzzy (anti-fuzzy) $H_{v}$-subgroups and investigating their properties.

\section{REFERENCES}

[1] M. Al-Tahan, B. Davvaz, Complex fuzzy $H_{v}$-subgroups of an $H_{v}$-group, submitted.

[2] P. Corsini, Join spaces, power sets, fuzzy sets, Proceedings of the Fifth Int. Congress of Algebraic Hyperstructures and Appl., 1993, Iasi, Romania, Hadronic Press, 1994.

[3] P. Corsini, A new connection between hypergroups and fuzzy sets, Southeast Asian Bull. Math. 27(2003), 221-229.

[4] P. Corsini and V. Leoreanu, Applications of Hyperstructures Theory, Advances in Mathematics, Kluwer Academic Publisher, 2003.

[5] B. Davvaz, Fuzzy $H_{v}$-groups, Fuzzy sets Syst. 101 (1999), 191-195.

[6] B. Davvaz, Polygroup Theory and Related Systems, World Scientific Publishing Co. Pte. Ltd., Hackensack, NJ, 2013. viii+200 pp.

[7] B. Davvaz and I. Cristea, Fuzzy Algebraic Hyperstructures- An introduction, Studies in Fuzziness and Soft Computing 321. Cham: Springer, 2015.

[8] F. Marty, Sur une generalization de la notion de group, In 8th Congress Math. Scandenaves, (1934), 45-49.

[9] A. Rosenfeld, Fuzzy groups, J. Math. Anal. Appl. 35 (1971), 512?17.

[10] D. Ramot, R. Milo, M. Friedman and A. Kandel, Complex fuzzy logic, IEEE trans. fuzzy syst. 10(2) (2002), 171-186.

[11] D. Ramot, M. Friedman, G. Langholz and A. Kandel, Complex fuzzy sets, IEEE trans. fuzzy syst. 11(4) (2003), $450-461$.

[12] T. Vougiouklis, Hyperstructures and Their Representations, Aviani editor. Hadronic Press, Palm Harbor, USA, 1994.

[13] T. Vougiouklis, A new class of hyperstructures, J. Combin. Inform. Syst. Sci. 20 (1995), $229 ? 35$.

[14] T. Vougiouklis, The fundamental relation in hyperrings. The general hyperfield, In: Proc of the 4th int. congress on algebraic hyperstructures and appl. (A.H.A 1990). World Sientific, Xanthi, Greece, pp 203-211.

[15] L. A. Zadeh, Fuzzy sets, Inf. Control 8 (1965), 338-353. 